\title{
Sarcopenia: ultrasound today, smartphones tomorrow?
}

\author{
Luca Maria Sconfienza ${ }^{1,2}$ (1)
}

Received: 25 September 2018 / Revised: 27 September 2018 / Accepted: 12 October 2018 / Published online: 12 November 2018

(C) European Society of Radiology 2018

Sarcopenia is a chronic disease characterized by a slow and progressive loss in the amount, composition, and function of skeletal muscle in the human body. The clinical implication of this condition is twofold: on the one hand, loss of muscle mass leads to muscle weakness, which in turns leads to reduced mobility and increased proneness to falls and fractures [1]; on the other hand, sarcopenia has been related to the occurrence of mortality, adverse events, and length of hospitalization in patients undergoing surgery for many pathologies [2]. This condition is gaining increasing importance, as it has been estimated that in 2016 sarcopenia was affecting around 20 million people in Europe, with an expected increase to more than 32 million people by $2045(+63.8 \%)$, corresponding to overall prevalence rates in the elderly of $20.2 \%$ and $22.3 \%$, respectively [3]. Thus, sarcopenia already represents a major medical and social challenge.

Currently, sarcopenia can be diagnosed with different modalities. Dual-energy X-ray absorptiometry (DXA) is the most common imaging-based method used in research and clinical practice, as it is simple, available, uses little amount of ionizing radiations, being also accurate and reproducible [4]. Thus, according to the European Working Group on Sarcopenia in Older People, DXA is considered a reliable tool to measure body composition and to obtain data about quantity and distribution of lean, fat, and mineral mass throughout the body [5]. On the other hand, computed tomography (CT) and magnetic resonance imaging (MRI) represent the reference standard for the diagnosis of sarcopenia, as both modalities allow

This comment refers to the article available at https://doi.org/10.1007/ s00330-018-5742-2.

Luca Maria Sconfienza

io@lucasconfienza.it

1 Unità Operativa di Radiologia Diagnostica ed Interventistica, IRCCS Istituto Ortopedico Galeazzi, Milano, Italy

2 Dipartimento di Scienze Biomediche per la Salute, Università degli Studi di Milano, Via Riccardo Galeazzi 4, 20161 Milan, Italy for precise evaluation of body composition and consistency of skeletal muscle on cross-sectional images. However, despite their high diagnostic value, the use of CT and MRI is limited by high burden of ionizing radiations or specific contraindications, respectively, by high costs, and by post-processing image analysis; thus, their use is generally restricted to research settings.

Despite being an excellent imaging modality in the evaluation of the musculoskeletal system [6], the use of ultrasound in the assessment of sarcopenic patients is relatively limited to the evaluation of thickness, cross-sectional area, and echo intensity of muscles. Consequently, no diagnostic algorithms currently include ultrasound as one of the possible diagnostic modalities for assessing sarcopenia. This is probably because most of those ultrasound parameters may be dependent on the operator and some of them are not able to fully differentiate minimal variations of muscle condition. In this setting, the pilot work by Sanabria et al [7] postulates the use of a new ultrasound-based method to identify sarcopenia in elderly subjects. This approach was recently used by the same group to calculate breast density [8]. They used a commercially available portable ultrasound system coupled with a simple plastic reflector to obtain the radiofrequency ultrasound data in the calf muscles of 10 elderly women and 11 healthy volunteers. Then, they developed a specific software capable to provide three-dimensional speed of sound calculation from raw ultrasound data. They found that this speed was significantly lower in elderly women than in young controls, yielding the remarkable diagnostic performance of $93.6 \%$. So far, then, this method has been proved to be excellent in differentiating women's age based on their muscular features, although it does not provide data on sarcopenia itself. I think that small sample size does not represent a limitation of this paper, as statistical significance was achieved for most of the presented comparisons. However, to understand if this method may really help in the diagnosis of sarcopenia, a further study comparing this tool with a more established technique to diagnose sarcopenia and with a more heterogeneous population is warranted. At any rate, these results are interesting and open the possibility of using commercial ultrasound systems to calculate sound 
velocities - similarly to what is done with shear-wave elastography, both for research purposes and as opportunistic screening. In fact, people generally seek for medical consultation only when they become symptomatic for a certain condition. Unfortunately, one of the most relevant problems in sarcopenia (as well as in other "silent" conditions, such as osteoporosis) is that otherwise-healthy subjects are generally not aware of their status. In this setting, the use of additional tools which can be applied to radiological examinations performed for other clinical conditions may represent a relevant innovation. On CT, this can be easier to do, as muscle attenuation has been proved to be a reliable parameter, while on MRI the use of specific sequences, such as 3-point Dixon, is generally preferred [9]. To my knowledge, nothing similar can be done on ultrasound, thus developing a simple and reliable tool which can be applied during ultrasound examinations performed for whatever reason may represent a real step forward.

Given that, it is evident sarcopenia is not only a medical condition but also a social and economic issue. Thus, on the one hand, future research should be aimed to find more advanced modalities able to give deep insights on the molecular mechanisms of this condition and enable monitoring even minimal variations over time. On the other hand, however, more commercial and easy-to-use tools should be made available to general population. Wearable devices are nowadays very diffused, most of them being aimed to monitor physical activity and parameters in subjects performing recreational-to-professional sport training. As an example, launching the Watch series 4, Apple claimed the possibility of generating an electrocardiogram similar to a single-lead electrocardiogram, thus providing potentially critical real-time data directly to the relevant physician and alert the subject in case of abnormalities [10]. Something similar could be developed also for sarcopenia, with elderly subjects who could continuously be monitored and have-for example - specific training activities suggested automatically by the relevant application on their smartphone according to the muscle mass loss detected by the wearable device. Is it a fantastic world? Probably not too much.

In conclusion, sarcopenia represents a very important societal challenge. Aside from the new technologies, imaging specialists should be aware that they should play a pivotal role in this setting and the new data presented by Sanabria and colleagues certainly goes in this direction.

Funding The authors state that this work has not received any funding.

\section{Compliance with ethical standards}

Guarantor The scientific guarantor of this publication is Luca Maria Sconfienza.

Conflict of interest The authors of this manuscript declare no relationships with any companies, whose products or services may be related to the subject matter of the article.

Statistics and biometry No complex statistical methods were necessary for this paper.

\section{References}

1. Messina C, Maffi G, Vitale JA, Ulivieri FM, Guglielmi G, Sconfienza LM (2018) Diagnostic imaging of osteoporosis and sarcopenia: a narrative review. Quant Imaging Med Surg 8:86-99

2. Sandini M, Pinotti E, Persico I, Picone D, Bellelli G, Gianotti L (2017) Systematic review and meta-analysis of frailty as a predictor of morbidity and mortality after major abdominal surgery. BJS Open 1:128-137

3. Ethgen O, Beaudart C, Buckinx F, Bruyère O, Reginster JY (2017) The future prevalence of sarcopenia in Europe: a claim for public health action. Calcif Tissue Int 100(3):229-234

4. Guglielmi G, Ponti F, Agostini M, Amadori M, Battista G, Bazzocchi A (2016) The role of DXA in sarcopenia. Aging Clin Exp Res 28:1047-1060

5. Cruz-Jentoft AJ, Baeyens JP, Bauer JM et al (2010) European Working Group on Sarcopenia in Older People. Sarcopenia: European consensus on definition and diagnosis: report of the European Working Group on Sarcopenia in Older People. Age Ageing 39:412-423

6. Sconfienza LM, Albano D, Allen G et al (2018) Clinical indications for musculoskeletal ultrasound updated in 2017 by European Society of Musculoskeletal Radiology (ESSR) consensus. Eur Radiol. https://doi.org/10.1007/s00330-018-5474-3

7. Sanabria SJ, Martini K, Freystätter G et al (2018) Speed of sound ultrasound: a pilot study on a novel technique to identify sarcopenia in seniors. Eur Radiol. https://doi.org/10.1007/s00330-018-5742-2

8. Sanabria SJ, Goksel O, Martini K et al (2018) Breast-density assessment with hand-held ultrasound: a novel biomarker to assess breast cancer risk and to tailor screening? Eur Radiol 28:3165-3317

9. Boutin RD, Yao L, Canter RJ, Lenchik L (2015) Sarcopenia: current concepts and imaging implications. AJR Am J Roentgenol 205:W255-W266

10. https://www.apple.com/apple-watch-series-4/health/ Accessed on 25 Sept 2018 